Supporting Information

S-1 


\section{A Novel Electrochemically Switchable, Flexible, Microporous Cloth that Selectively Captures, Releases and Concentrates Intact Extracellular Vesicles}

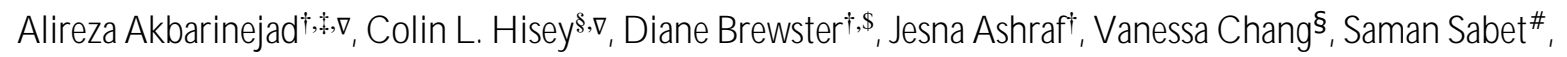

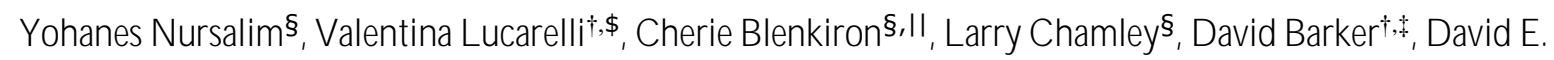
Williams ${ }^{*},+*$, Clive W. Evans ${ }^{*}, \dagger$, Jadranka T ravas-Sejdic ${ }^{*},+,+$

+Polymer Biointerface C entre, School of C hemical Sciences, The U niversity of Auckland, Auckland 1010, N ew Zealand

$\ddagger$ The M acDiarmid I nstitute of Advanced M aterials and N anotechnology, Wellington 6140, N ew Zealand

$\S \mathrm{H}$ ub for Extracellular Vesicles I nvestigations ( $\mathrm{HEVI}$ ) , D epartment of O bstetrics and G ynecology, The U niversity of Auckland, Auckland 1023, N ew Zealand

$\$ T$ he N ew Zealand Institute for Plant and Food Research Limited, Private Bag 92169, A uckland, 1142 , N ew Zealand

|lD epartment of M olecular M edicine and Pathology, T he U niversity of A uckland, Auckland, N ew Zealand

\#School of Chemical Sciences, T he U niversity of Auckland, Auckland 1010, N ew Zealand

\section{Corresponding Authors}

*.t.travas-sejdic@auckland.ac.nz

*c.evans@auckland.ac.nz

*david.williams@auckland.ac.nz 


\section{Contents}

Figure S1. Sulfonation of SEBS polymer. (a) Chemical structure of SEBS and sulfonated SEBS (SSEBS). (b) Weight percentage of different elements in SEBS and SSEBS measured by CHNS elemental analysis.

Figure S2. Schematic illustration of the electrospinning setup used in this work

Figure S3. Raman spectrum of SSEBS-PEDOT cloth (excitation wavelength $532 \mathrm{~nm}$ ). The presence of PEDOT characteristic peaks at 437, 568 and 988 (oxyethylene ring deformation); 698 (symmetric C-S-C stretching); 1095 (C-O-C stretching); 1264 (C $\alpha-\mathrm{C} \alpha) ; 1364$ (C $\beta-\mathrm{C} \beta$ stretching); 1431 (symmetric $\mathrm{C} \alpha=\mathrm{C} \beta$ stretching); 1509 (asymmetric $\mathrm{C} \alpha=\mathrm{C} \beta$ stretching); confirm the successful coating of PEDOT onto the fibers. ${ }^{1-3}$.....

Figure S4. XPS spectra of (a) SSEBS and (b) SSEBS-PEDOT cloths. The high S/C elemental ratio of SSEBS-PEDOT cloth (0.07) as compared to SSEBS (0.005) confirms the successful PEDOT coating.

Figure S5. SEM images of the SSEBS-PEDOT electrospun cloth incubated into $\mathrm{HAuCl}_{4}$ solution, showing the coating by Au particles without using any reducing agent, (a) 200 and (b) 500 magnification.

Figure S6. SEM images of the SSEBS-PEDOT electrospun cloth incubated into $\mathrm{HAuCl}_{4}$ solution followed by trisodium citrate treatment at different magnifications of (a) 200 and (b) 500.......... 7

Figure S7. UV-vis spectra of $\mathrm{HAuCl}_{4}(1 \mathrm{mM})$ aqueous solutions before and after the incubation with SSEBS-PEDOT cloth.

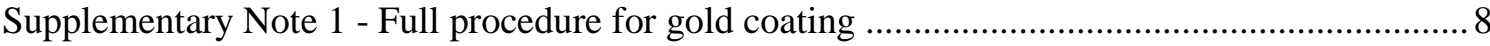

Figure S8. Photographs of the bare SSEBS and PEDOT-embedded electrospun cloths before $\mathrm{Au}$ coating and after Au coating without and with $N, N$-dimethylethylenediamine treatment. 8

Figure S9. UV-vis spectra of $\mathrm{HAuCl}_{4}(1 \mathrm{mM})$ aqueous solutions before and after the incubation of different substrates

Figure S10. Size distribution profile of the gold nanoparticles (a) and micro-flowers (b) deposited on SSEBS-PEDOT cloth

Figure S11. Stability of the electrospun cloth under electrochemical potential stimulation. (a, b) SEM images of SSEBS-PEDOT-Au cloth at different magnifications after exposing to electrochemical potential $(-1.2 \mathrm{~V}$ vs. $\mathrm{Ag} / \mathrm{AgCl})$ in PBS for $10 \mathrm{~min}$. (c) The changes in the conductivity level of SSEBS-PEDOT-Au cloth upon stimulation at $-1.2 \mathrm{~V}$ vs. $\mathrm{Ag} / \mathrm{AgCl}$ for 0-40 $\min (\mathrm{n}=3) . * * \mathrm{p}<0.01$.

Supplementary Note 2 - Full procedure for 2ME capture/release

Figure S12. The fluorescence emission $\left(\lambda_{\mathrm{ex}}=330 \mathrm{~nm}\right)$ and excitation $\left(\lambda_{\mathrm{em}}=450 \mathrm{~nm}\right)$ spectra of the $o$-phthalaldehyde /alanine solution $(1 \mathrm{mM}$ each) in the presence of $110 \mathrm{nM} 2 \mathrm{ME}$.

Figure S13. The fluorescence emission spectra of o-phthalaldehyde/alanine solution $\left(\lambda_{\mathrm{ex}}=330\right.$ $\mathrm{nm})$ in the presence of $0-110 \mathrm{nM} 2 \mathrm{ME}$, the inset shows the corresponding calibration plot $(\mathrm{n}=$ 3).

Figure S14. Schematic illustration of the capture and electrochemical release of 2ME on SSEBSPEDOT-Au cloth.

Figure S15. (a) The fluorescence emission spectra of $\left(\lambda_{\mathrm{ex}}=330 \mathrm{~nm}\right)$ : (a) $0.1 \mathrm{M}$ of $\mathrm{LiClO}_{4}$ solutions incubated with $2 \mathrm{ME}$-coated cloth without potential stimulation (black line), the first potential stimulation at $-1.2 \mathrm{~V}$ for $5 \mathrm{~min}$ (red line) and the second potential stimulation at $-1.2 \mathrm{~V}$ for $5 \mathrm{~min}$ (blue line). (b) $1 \mathrm{mM}$ o-phthalaldehyde/alanine in $0.1 \mathrm{M}$ of $\mathrm{LiClO}_{4}$ aqueous solution 
(orange line), $5 \mathrm{~mL}$ of $0.1 \mathrm{M} \mathrm{LiClO}_{4}$ aqueous solution incubated with a $1 \times 1 \mathrm{~cm} \mathrm{SSEBS-}$

PEDOT-Au cloth for $1 \mathrm{~h}$ (blue line) and $5 \mathrm{~mL}$ of $0.1 \mathrm{M} \mathrm{LiClO}_{4}$ aqueous solution incubated with a $1 \times 1 \mathrm{~cm}$ SSEBS-PEDOT-Au cloth for $1 \mathrm{~h}$ plus $1 \mathrm{mM}$ o-phthalaldehyde/alanine.

Figure S16. EV purification on qEV columns. Size distribution profile of different EV fractions ( $5 \mathrm{~mL}$ fractions), obtained by NanoSight.

Figure S17. EV and protein concentration profiles of $5 \mathrm{~mL}$ MCF-7 in fractions separated using qEV10 columns..

Figure S18. TEM images of the MCF-7 EVs pre capture/release. The bar in all of the TEM images, except the central image, is $200 \mathrm{~nm}$.

Figure S19. Normalized NTA count of MCF-7 and HDFa EVs from 8x T175s $(n=3)$. *** $<<$ 0.001 ......

Figure S20. Experimental setup used for EV capture/release. (a) Schematic view. Photograph of the 3D printed capture/release cell without (b) and with the electrodes connected (c).

Figure S21. Confocal imaging analysis of control cloths. (a) Confocal image of the electrospun cloth with no aptamer, incubated with MCF-7 EVs. (b) Confocal image of the CD63 aptamerfunctionalized cloth incubated with PKH67 fluorescent dye.

Figure S22. TEM images of the MCF-7 EVs after capture/release process using CD63 aptamerfunctionalized cloth. The bar in all of the TEM images, except the central image, is $200 \mathrm{~nm}$.... 16

Figure S23. Coomassie blue-stained membranes used for CD63 (a) and CD81 (b) Western blots.

Figure S24. $\zeta$ potential values of the MCF-7 EVs before and after capture/release on the CD63

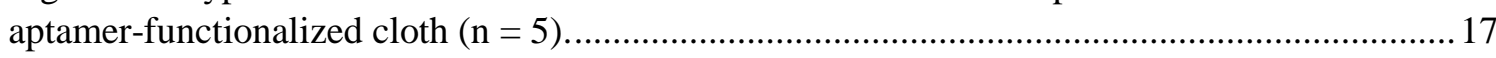

References 

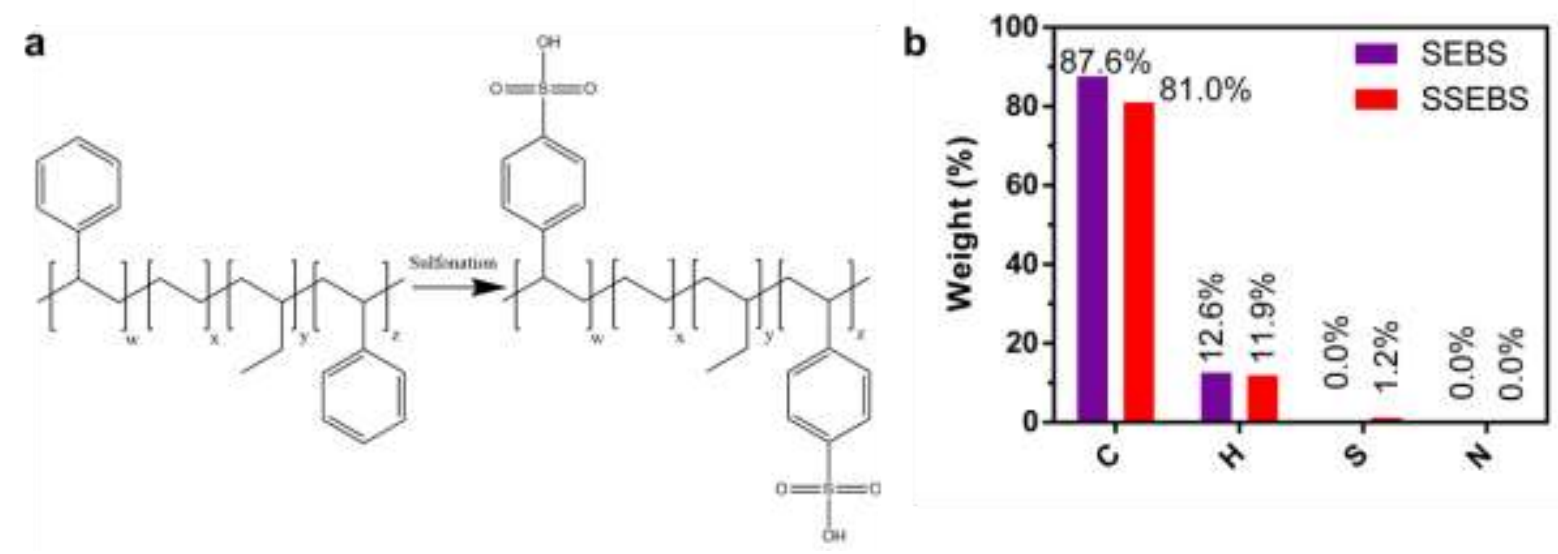

Figure S1. Sulfonation of SEBS polymer. (a) Chemical structure of SEBS and sulfonated SEBS (SSEBS). (b) Weight percentage of different elements in SEBS and SSEBS measured by CHNS elemental analysis.

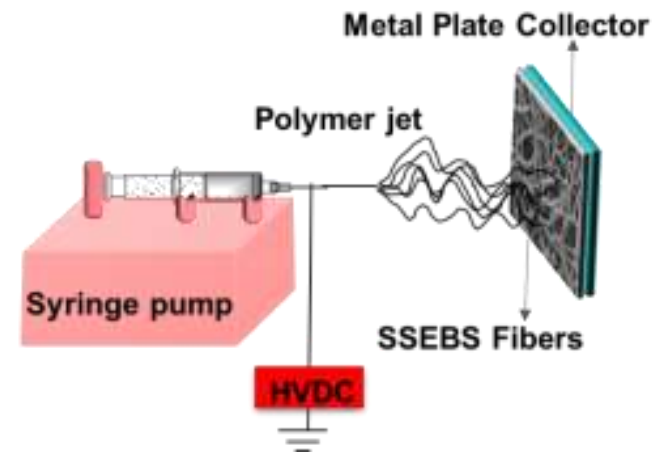

Figure S2. Schematic illustration of the electrospinning setup used in this work. 


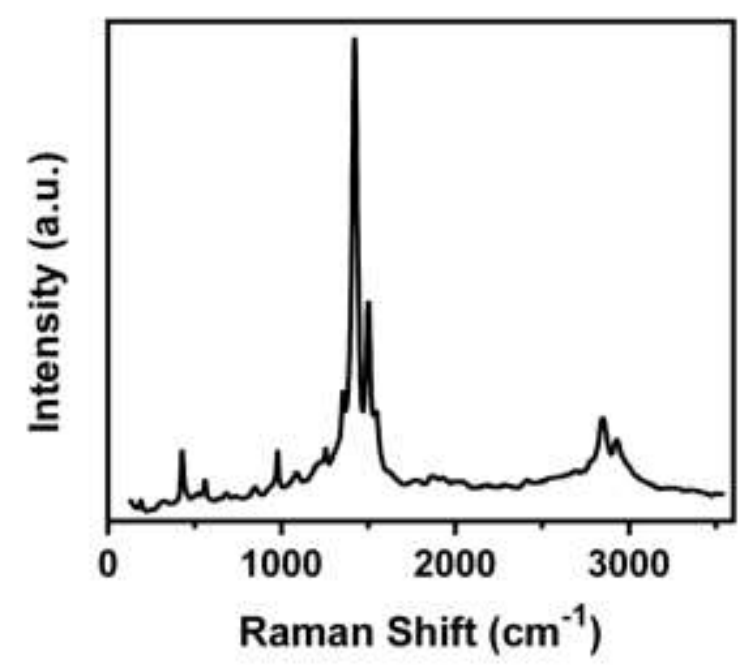

Figure S3. Raman spectrum of SSEBS-PEDOT cloth (excitation wavelength $532 \mathrm{~nm}$ ). The presence of PEDOT characteristic peaks at $437 \mathrm{~cm}^{-1}, 568 \mathrm{~cm}^{-1}$ and $988 \mathrm{~cm}^{-1}$ (oxyethylene ring deformation); 698 $\mathrm{cm}^{-1}$ (symmetric C-S-C stretching); $1095 \mathrm{~cm}^{-1}$ (C-O-C stretching); $1264 \mathrm{~cm}^{-1}(\mathrm{C} \alpha-\mathrm{C} \alpha) ; 1364 \mathrm{~cm}^{-1}$ (C $\beta-$ $\mathrm{C} \beta$ stretching); $1431 \mathrm{~cm}^{-1}$ (symmetric $\mathrm{C} \alpha=\mathrm{C} \beta$ stretching); $1509 \mathrm{~cm}^{-1}$ (asymmetric $\mathrm{C} \alpha=\mathrm{C} \beta$ stretching); confirm the successful coating of PEDOT onto the fibers. ${ }^{1-3}$
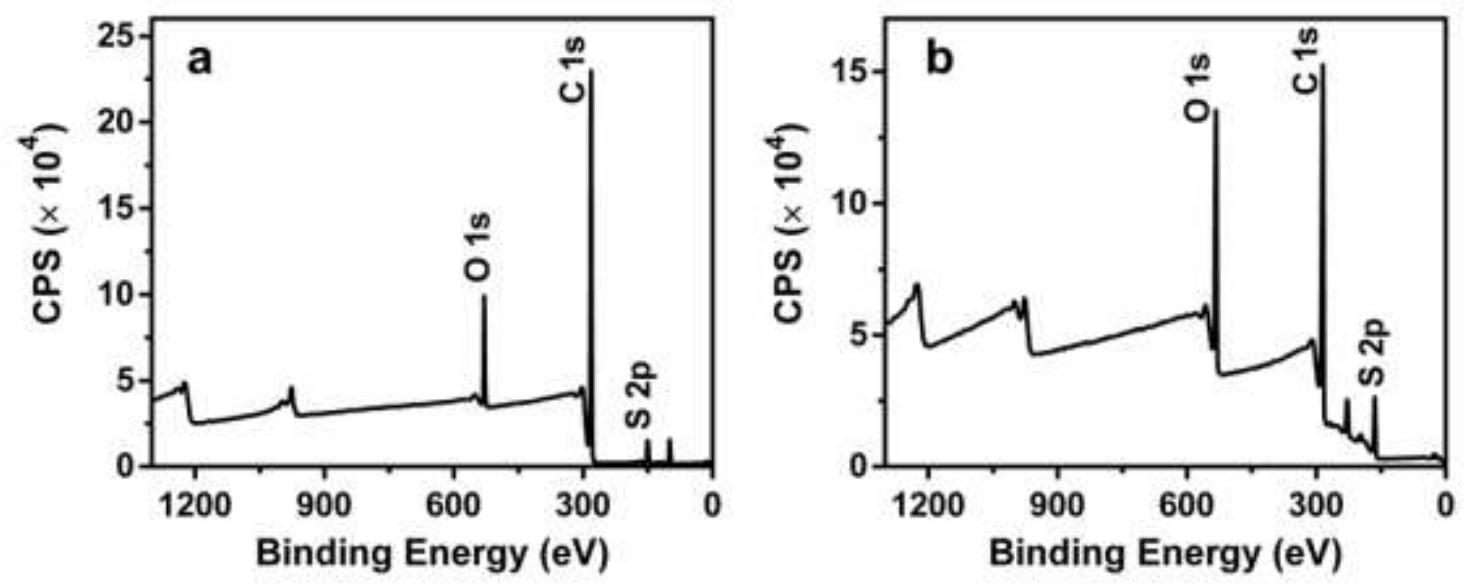

Figure S4. XPS spectra of (a) SSEBS and (b) SSEBS-PEDOT cloths. The high S/C elemental ratio of SSEBS-PEDOT cloth (0.07) as compared to SSEBS (0.005) confirms the successful PEDOT coating. 

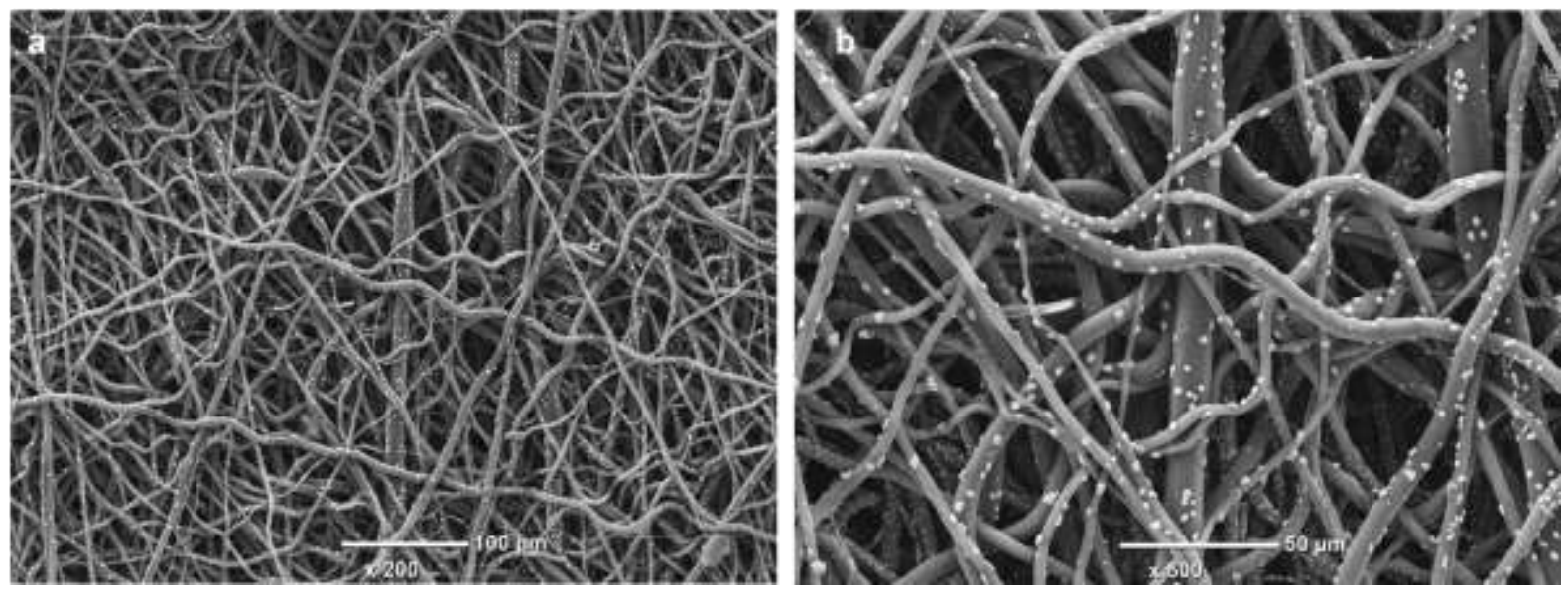

Figure S5. SEM images of the SSEBS-PEDOT electrospun cloth incubated into $\mathrm{HAuCl}_{4}$ solution, showing the coating by $\mathrm{Au}$ particles without using any reducing agent, (a) 200 and (b) 500 magnification.

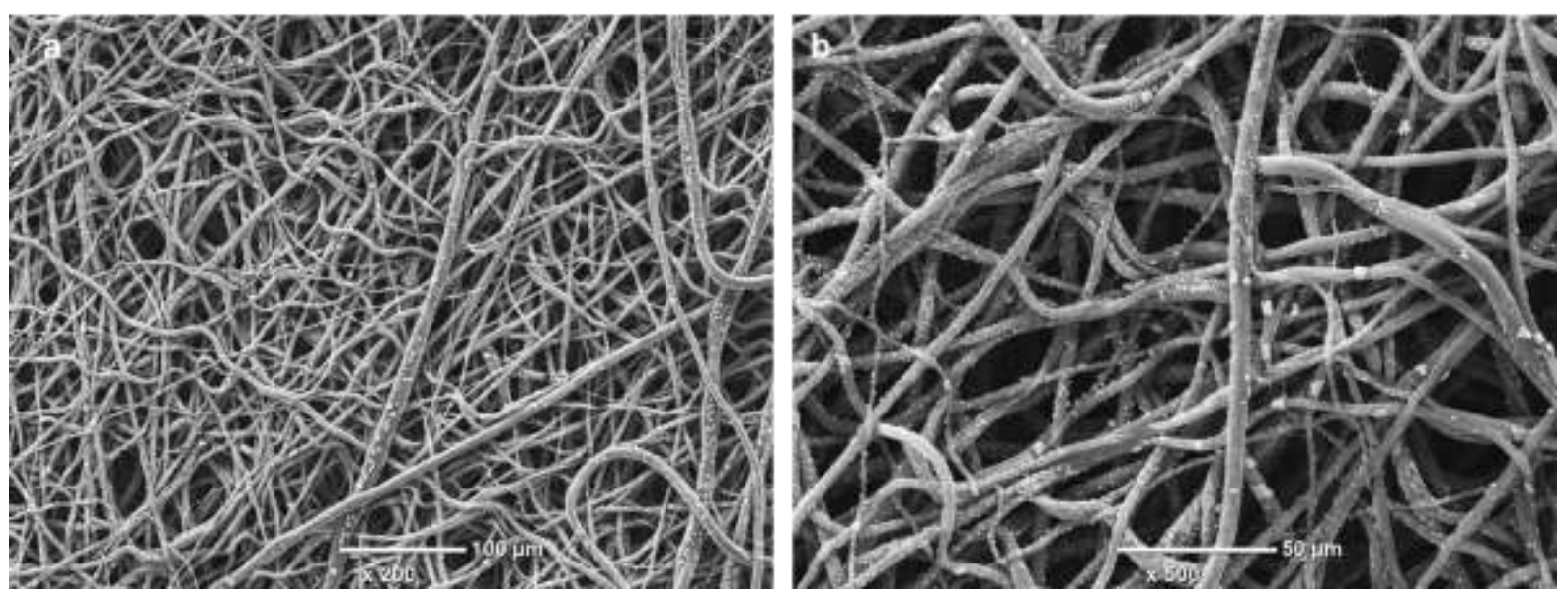

Figure S6. SEM images of the SSEBS-PEDOT electrospun cloth incubated into $\mathrm{HAuCl}_{4}$ solution followed by trisodium citrate treatment at different magnifications of (a) 200 and (b) 500 .

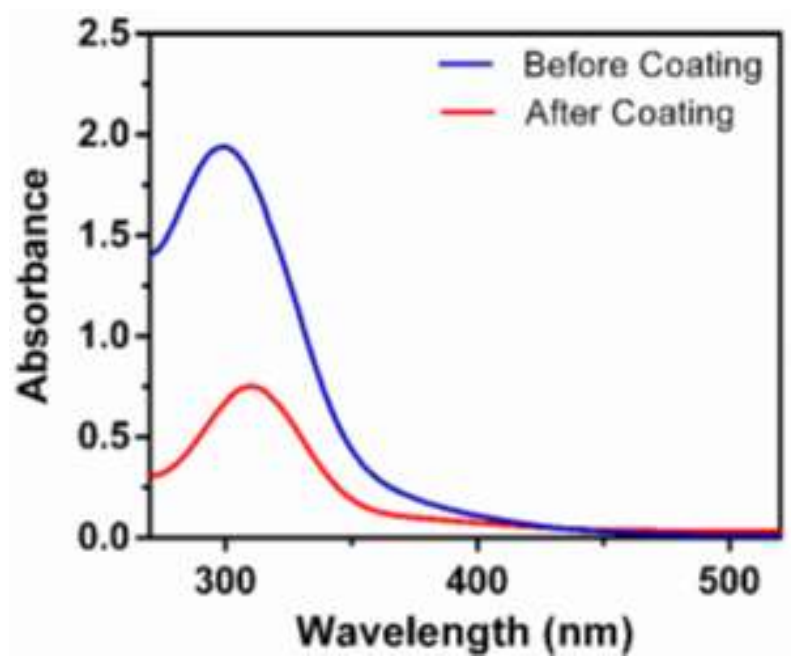

Figure S7. UV-vis spectra of $\mathrm{HAuCl}_{4}(1 \mathrm{mM})$ aqueous solutions before and after the incubation with SSEBS-PEDOT cloth. 


\section{Supplementary Note 1 - Full procedure for gold coating}

We performed experiments of Au coating on a number of electrospun mats including: SSEBS, SSEBS treated with $N, N$-dimethylethylenediamine, SSEBS-PEDOT and SSEBS-PEDOT treated with $N, N$-dimethylethylenediamine. According to the photographs of the electrospun cloths, as shown in Figure S8 and UV-vis spectra presented in Figure S9, the Au coating on the SSEBS mat was not satisfactory, which was presumed due to the poor interaction of $\mathrm{AuCl}_{4}{ }^{-}$ ions with the SSEBS fiber surface.

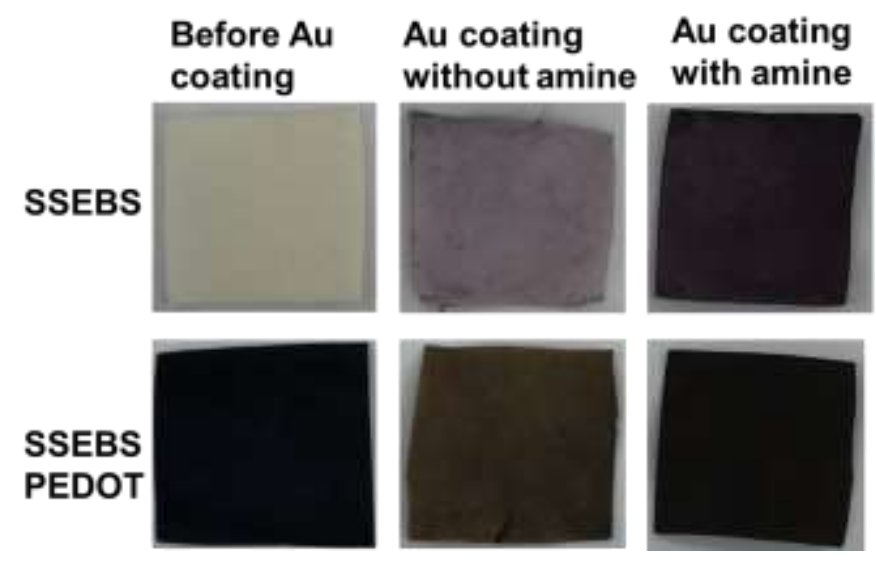

Figure S8. Photographs of the bare SSEBS and PEDOT-embedded electrospun cloths before $\mathrm{Au}$ coating and after Au coating without and with $N, N$-dimethylethylenediamine treatment.

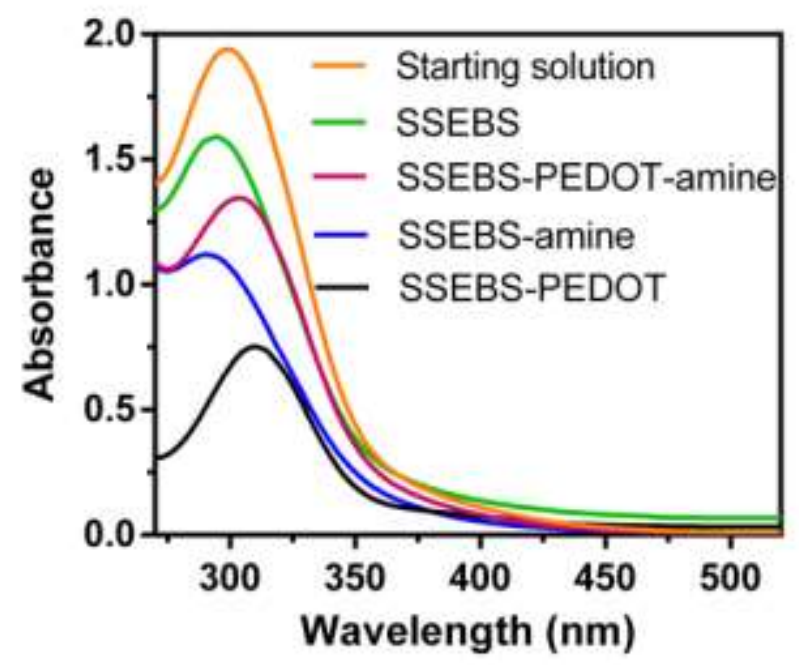

Figure S9. UV-vis spectra of $\mathrm{HAuCl}_{4}(1 \mathrm{mM})$ aqueous solutions before and after the incubation of different substrates.

On the other hand, Au coating on the SSEBS mat was much more effective when the fibers were treated with $\mathrm{N}, \mathrm{N}$-dimethylethylenediamine. We presume that the diamine interacts with 
the SSEBS backbone through some of the present amine groups, while the residual amine group can be available to complex $\mathrm{AuCl}_{4}^{-}$anions via electrostatic interactions. The efficient $\mathrm{Au}$ coating of diamine-treated substrate was evident from the strong purple color of the substrate and considerable decrease in UV-vis absorption of gold salt solution. Despite the efficient $\mathrm{Au}$ coating of SSEBS fibers, they did not show electrical conductivity, which would hamper their performance as a capture/release cloth. To alleviate this limitation, the Au coating procedure was carried out on bare and diamine functionalized SSEBS-PEDOT mat. As the photographs and UV-vis spectra show, the existence of PEDOT polymer facilitated the process of $\mathrm{Au}$ coating. Surprisingly, the coating of diamine-treated SSEBS-PEDOT mat with Au was not as good as the bare SSEBS-PEDOT mat, which could be attributed to deactivation of polarons and bipolarons by $\mathrm{N}, \mathrm{N}$-dimethylethylenediamine treatment. It is well known that amine groups can strongly interact with polarons and bipolarons through a donor-acceptor process, in which electron donating amine molecules interact with the positive charge in the conjugated polymer, according to the following equation: ${ }^{4}$

$\mathrm{PEDOT}^{+}+\mathrm{RNH}_{2} \rightarrow(\mathrm{PEDOT}: \mathrm{RNH} 2)^{+}$

Therefore, we propose that the interaction of amine molecules with polarons reduces the affinity of PEDOT toward $\mathrm{AuCl}_{4}{ }^{-}$, resulting in a decreased amount of Au particles deposited.

The SEM images of the SSEBS-PEDOT-Au mats (Figure 2f) show two types of morphologies of gold particles on the electrospun fibers: Au nanoparticles (NPs) and 'micro-flowers'. The gold nanoparticles and micro-flowers have size distributions from 10-140 nm and 0.6-1.7 $\mu \mathrm{m}$ with an average diameter of $59 \mathrm{~nm}$ and $1.0 \mu \mathrm{m}$, respectively (Figure S10). The formation of Au nano- and microstructures could be attributed to the initial deposition of Au NPs and the subsequent crystallization of $\mathrm{Au}$ microstructures (called here 'micro-flowers') due to inhomogeneous diffusion of gold salt onto the Au NPs. 5, 6 

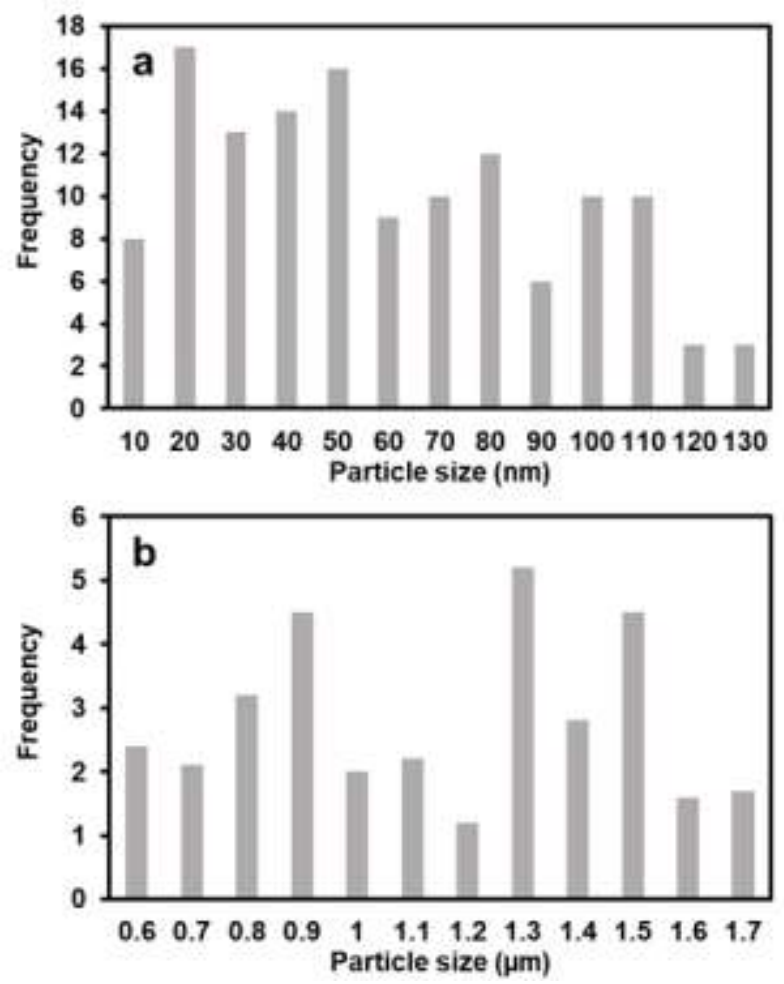

Figure S10. Size distribution profile of the gold nanoparticles (a) and micro-flowers (b) deposited on SSEBS-PEDOT cloth.
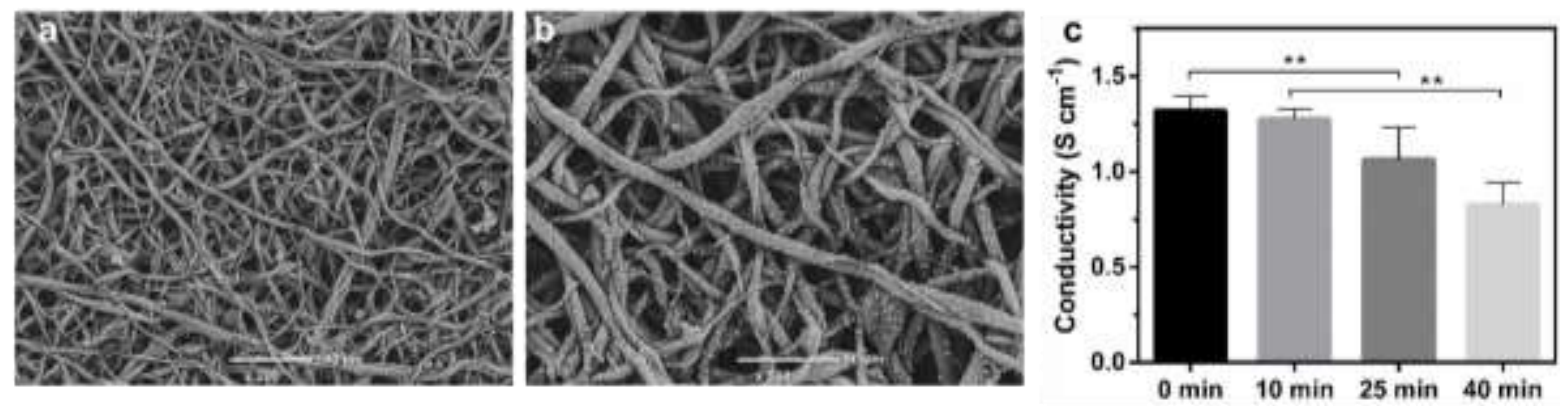

Figure S11. Stability of the electrospun cloth under electrochemical potential stimulation. (a, b) SEM images of SSEBS-PEDOT-Au cloth at different magnifications after exposing to electrochemical potential (-1.2 V vs. $\mathrm{Ag} / \mathrm{AgCl}$ ) in PBS for $10 \mathrm{~min}$. (c) The changes in the conductivity level of SSEBSPEDOT-Au cloth upon stimulation at $-1.2 \mathrm{~V}$ vs. $\mathrm{Ag} / \mathrm{AgCl}$ for $0-40 \mathrm{~min}(\mathrm{n}=3)$. **p $<0.01$.

\section{Supplementary Note 2 - Full procedure for $2 \mathrm{ME}$ capture/release}

We used 2-mercaptoethanol (2ME) as a model compound to confirm the capture and electrochemical release ability of the substrate. The principle of the capture of this model compound is based on the formation of a fluorescent adduct in solution between $2 \mathrm{ME}, o-$ phthaldehyde and alanine. ${ }^{7,8}$ The fluorescence emission and excitation spectra of the ophthalaldehyde/alanine solution in the presence of $110 \mathrm{nM} 2 \mathrm{ME}$ is presented in Figure S12. The maximum excitation and emission wavelengths of the formed fluorescent adduct were found to be 330 and $450 \mathrm{~nm}$, respectively. The concentration of the formed flurophore in the 
solution was measured upon addition of a range of concentrations of $2 \mathrm{ME}$ and a calibration graph was obtained (Figure S13). When the SSEBS-PEDOT-Au fiber mat was used, a potential of $-1.2 \mathrm{~V}$ (vs. $\mathrm{Ag} / \mathrm{AgCl}$ ) was applied to the conductive mat (in aqueous $0.1 \mathrm{M} \mathrm{LiClO}_{4}$ ) for 5 min to electrochemically release $2 \mathrm{ME}$ bound to the cloth, through the cleavage of gold-thiol bond (Figure S14). A clear and strong fluorescence emission of the solution was detected (Figure S15a) demonstrating sucessful electrochemical release of $2 \mathrm{ME}$ from the cloth. The fluorescence measurements on a number of control samples confirm that the observed signal is due to the flourescent adduct of $o$-phthaldehyde, alanine and 2ME (Figure S15b). Assuming that the $2 \mathrm{ME}$ forms a close-packed self-assembled layer on gold, and that the release efficiency is $100 \%$, the detected $2 \mathrm{ME}$ concentration in the released solution leads to an estimate of the maximum number of possibly bound $2 \mathrm{ME}$ molecules of $43.8 \pm 6.5 \mathrm{pmol} / \mathrm{mg}$ of fibre mat.

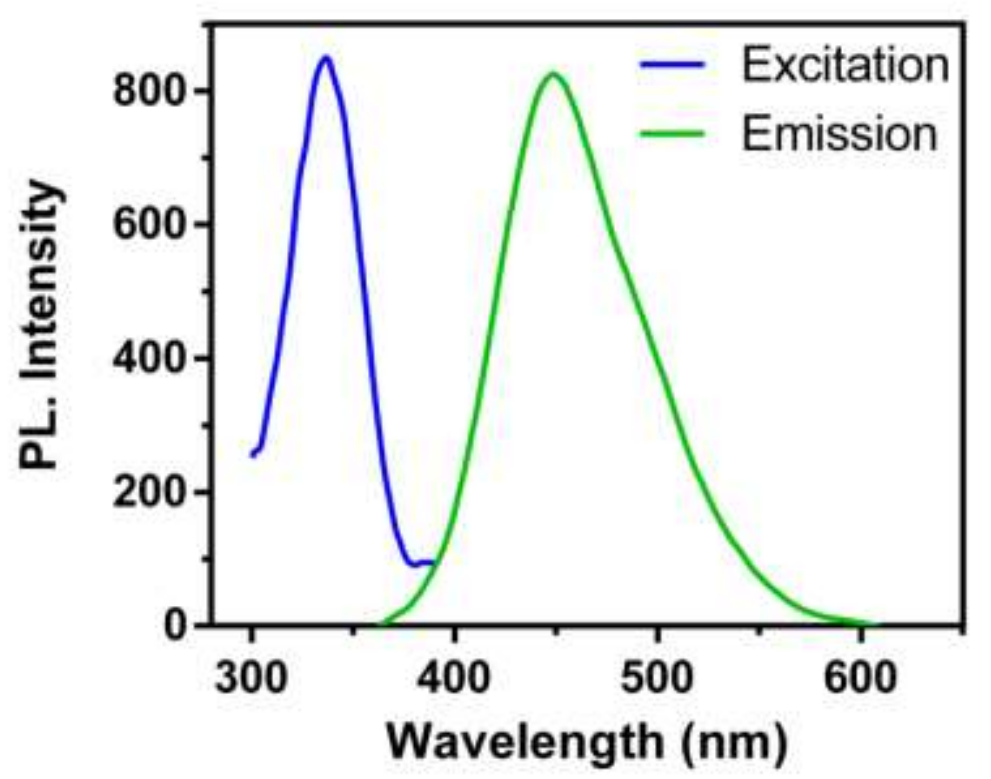

Figure S12. The fluorescence emission $\left(\lambda_{\mathrm{ex}}=330 \mathrm{~nm}\right)$ and excitation $\left(\lambda_{\mathrm{em}}=450 \mathrm{~nm}\right)$ spectra of the $o$ phthalaldehyde /alanine solution (1 mM each) in the presence of $110 \mathrm{nM} 2 \mathrm{ME}$. 


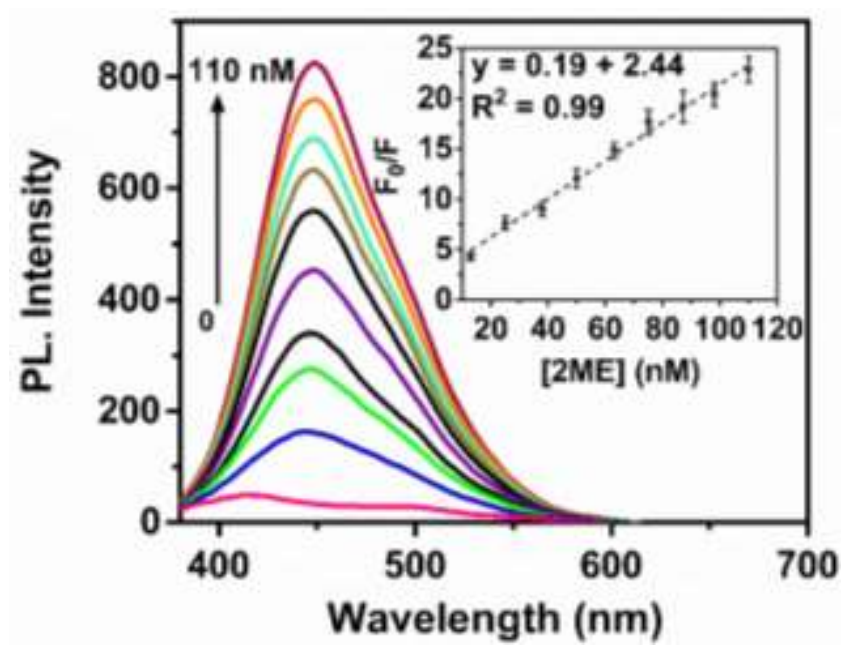

Figure S13. The fluorescence emission spectra of o-phthalaldehyde/alanine solution $\left(\lambda_{\mathrm{ex}}=330 \mathrm{~nm}\right)$ in the presence of $0-110 \mathrm{nM} 2 \mathrm{ME}$, the inset shows the corresponding calibration plot $(\mathrm{n}=3)$.

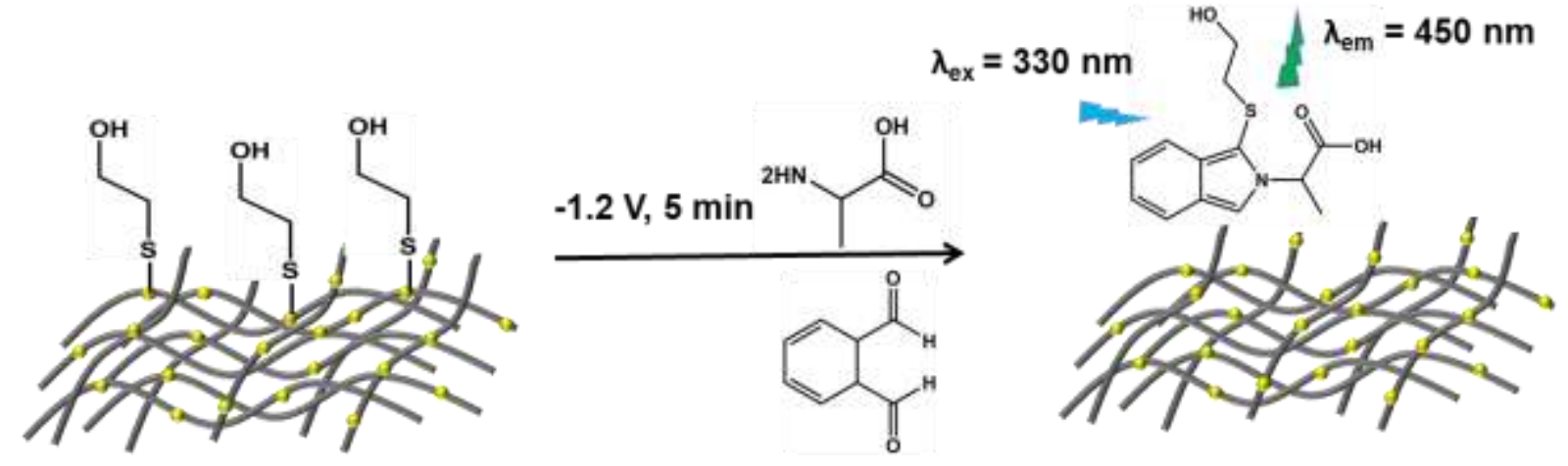

Figure S14. Schematic illustration of the capture and electrochemical release of 2ME on SSEBSPEDOT-Au cloth. 
a

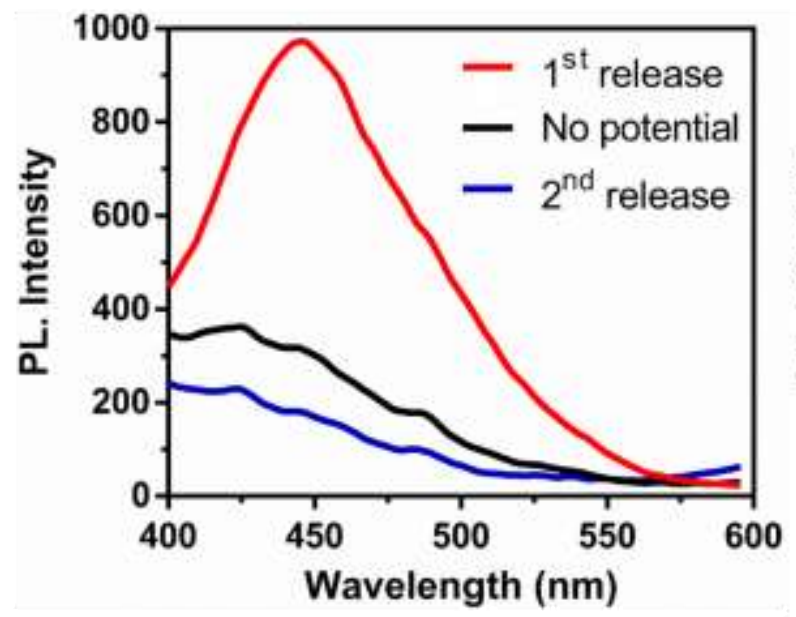

b

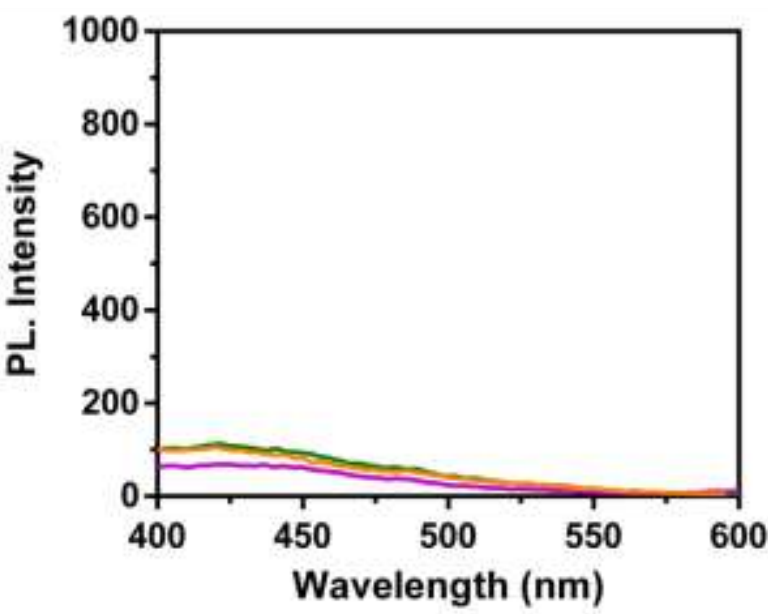

Figure S15. (a) The fluorescence emission spectra of $\left(\lambda_{\mathrm{ex}}=330 \mathrm{~nm}\right)$ : (a) $0.1 \mathrm{M}$ of $\mathrm{LiClO}_{4}$ solutions incubated with $2 \mathrm{ME}$-coated cloth without potential stimulation (black line), the first potential stimulation at $-1.2 \mathrm{~V}$ for $5 \mathrm{~min}$ (red line) and the second potential stimulation at $-1.2 \mathrm{~V}$ for $5 \mathrm{~min}$ (blue line). (b) $1 \mathrm{mM}$ o-phthalaldehyde/alanine in $0.1 \mathrm{M}$ of $\mathrm{LiClO}_{4}$ aqueous solution (orange line), $5 \mathrm{~mL}$ of $0.1 \mathrm{M} \mathrm{LiClO}_{4}$ aqueous solution incubated with a $1 \times 1 \mathrm{~cm}$ SSEBS-PEDOT-Au cloth for $1 \mathrm{~h}$ (blue line) and $5 \mathrm{~mL}$ of $0.1 \mathrm{M} \mathrm{LiClO}_{4}$ aqueous solution incubated with a $1 \times 1 \mathrm{~cm}$ SSEBS-PEDOT-Au cloth for 1 h plus $1 \mathrm{mM}$ o-phthalaldehyde/alanine.

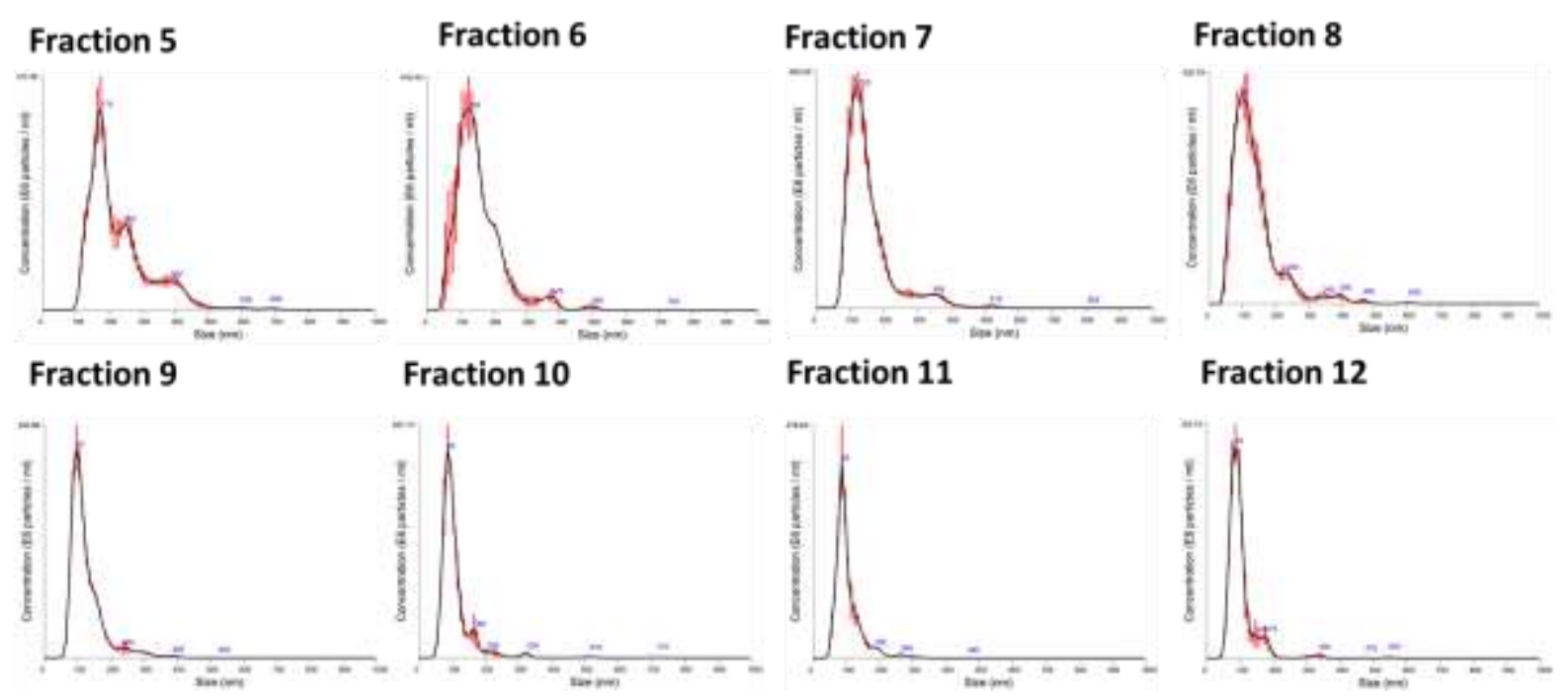

Figure S16. EV purification on qEV columns. Size distribution profile of different EV fractions (5 mL fractions), obtained by NanoSight. 


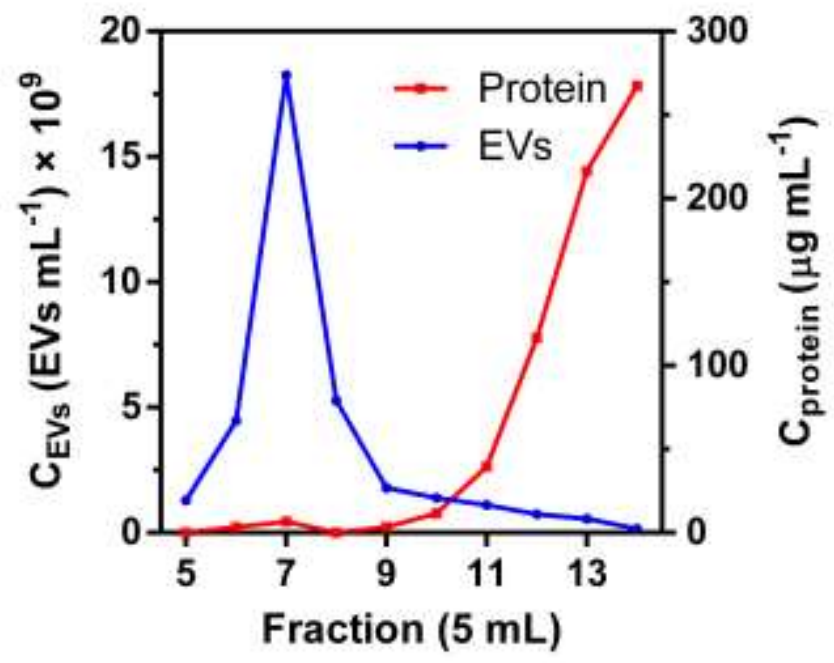

Figure S17. EV and protein concentration profiles of $5 \mathrm{~mL}$ MCF-7 in fractions separated using qEV10 columns.

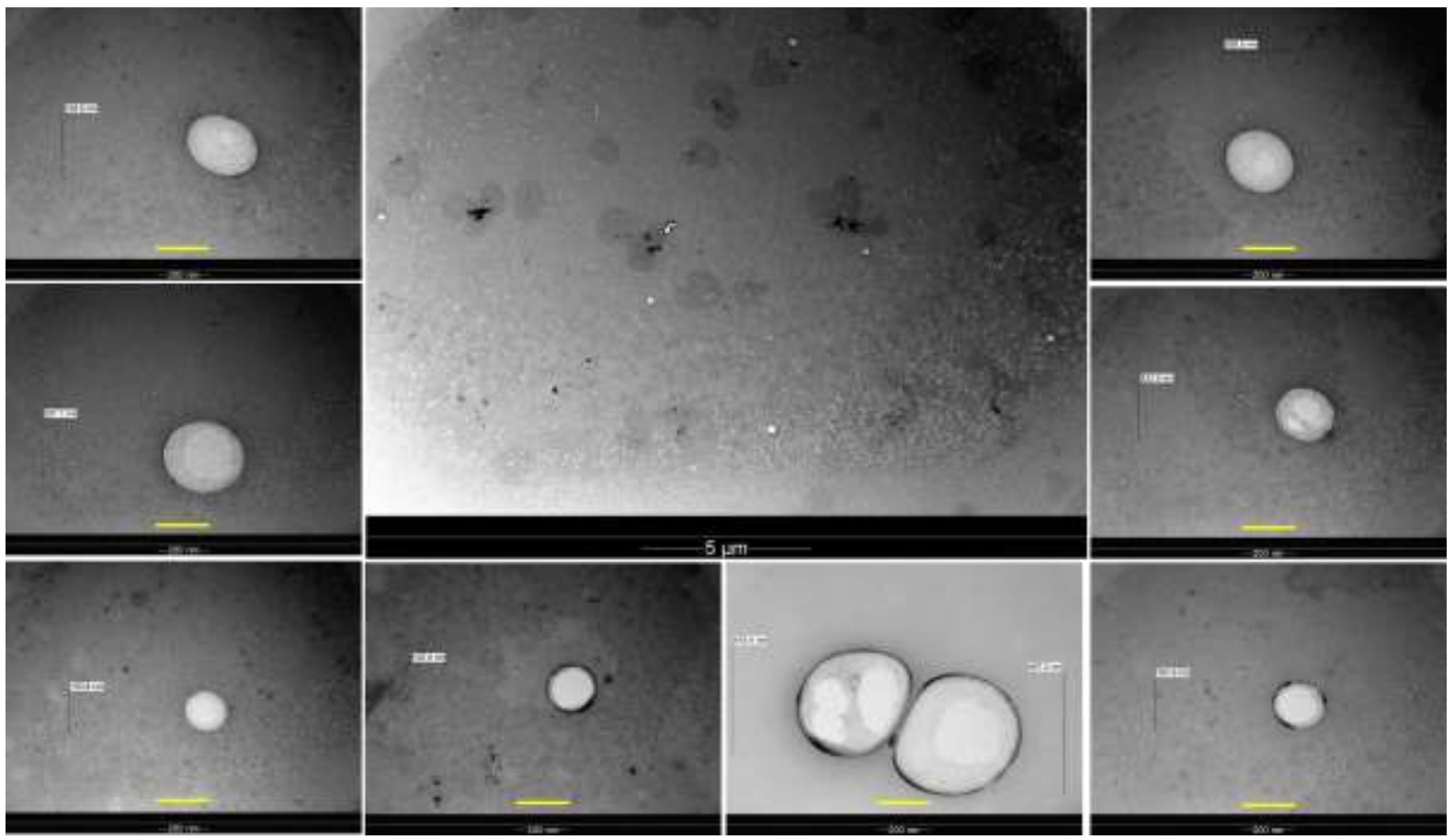

Figure S18. TEM images of the MCF-7 EVs pre capture/release. The bar in all of the TEM images, except the central image, is $200 \mathrm{~nm}$. 


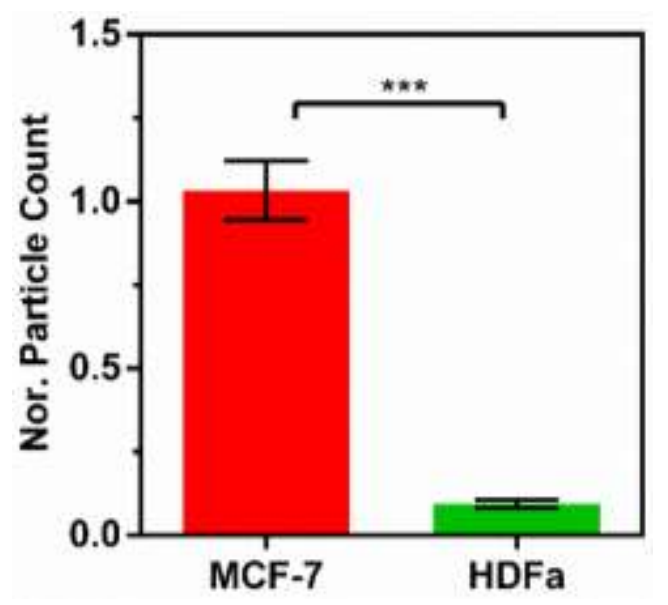

Figure S19. Normalized NTA count of MCF-7 and HDFa EVs from 8x T175s $(\mathrm{n}=3)$. *** $\mathrm{p}<0.001$.

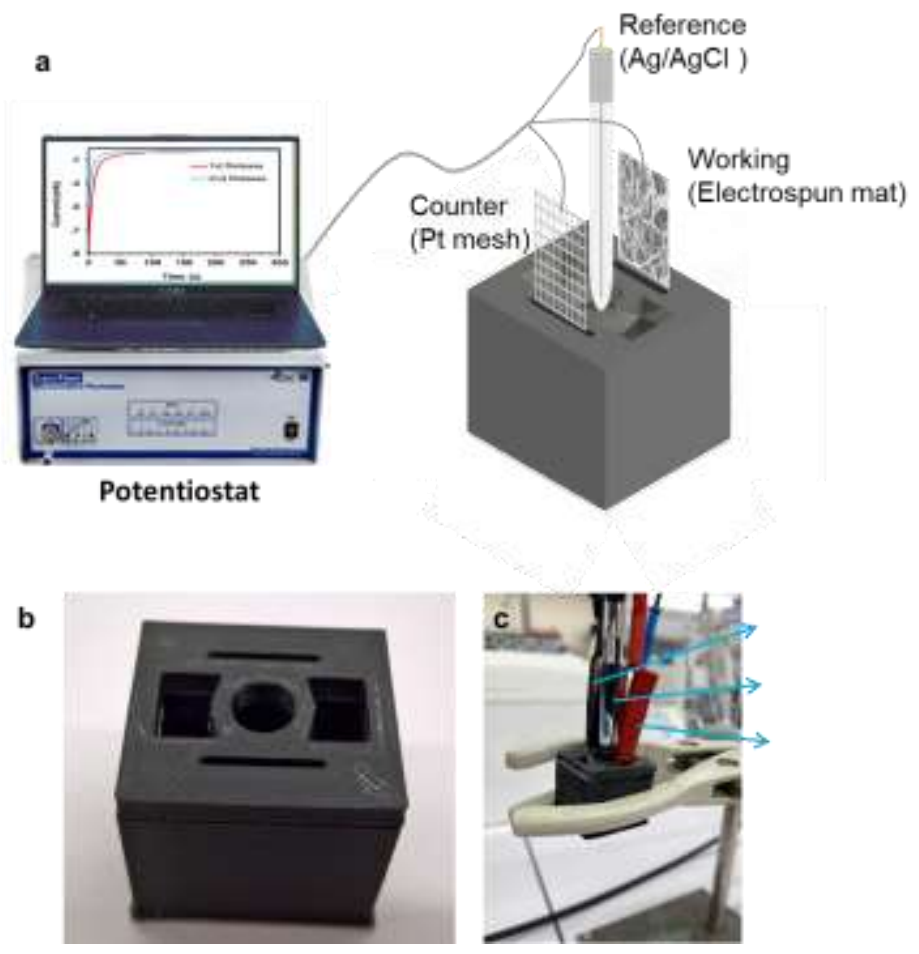

Figure S20. Experimental setup used for EV capture/release. (a) Schematic view. Photograph of the 3D printed capture/release cell without (b) and with the electrodes connected (c). 


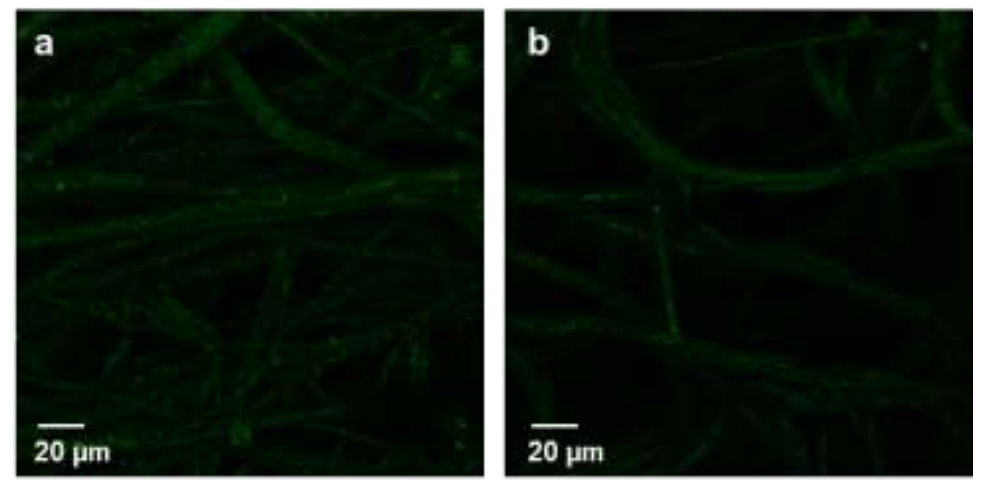

Figure S21. Confocal imaging analysis of control cloths. (a) Confocal image of the electrospun cloth with no aptamer, incubated with MCF-7 EVs. (b) Confocal image of the CD63 aptamer-functionalized cloth incubated with PKH67 fluorescent dye.
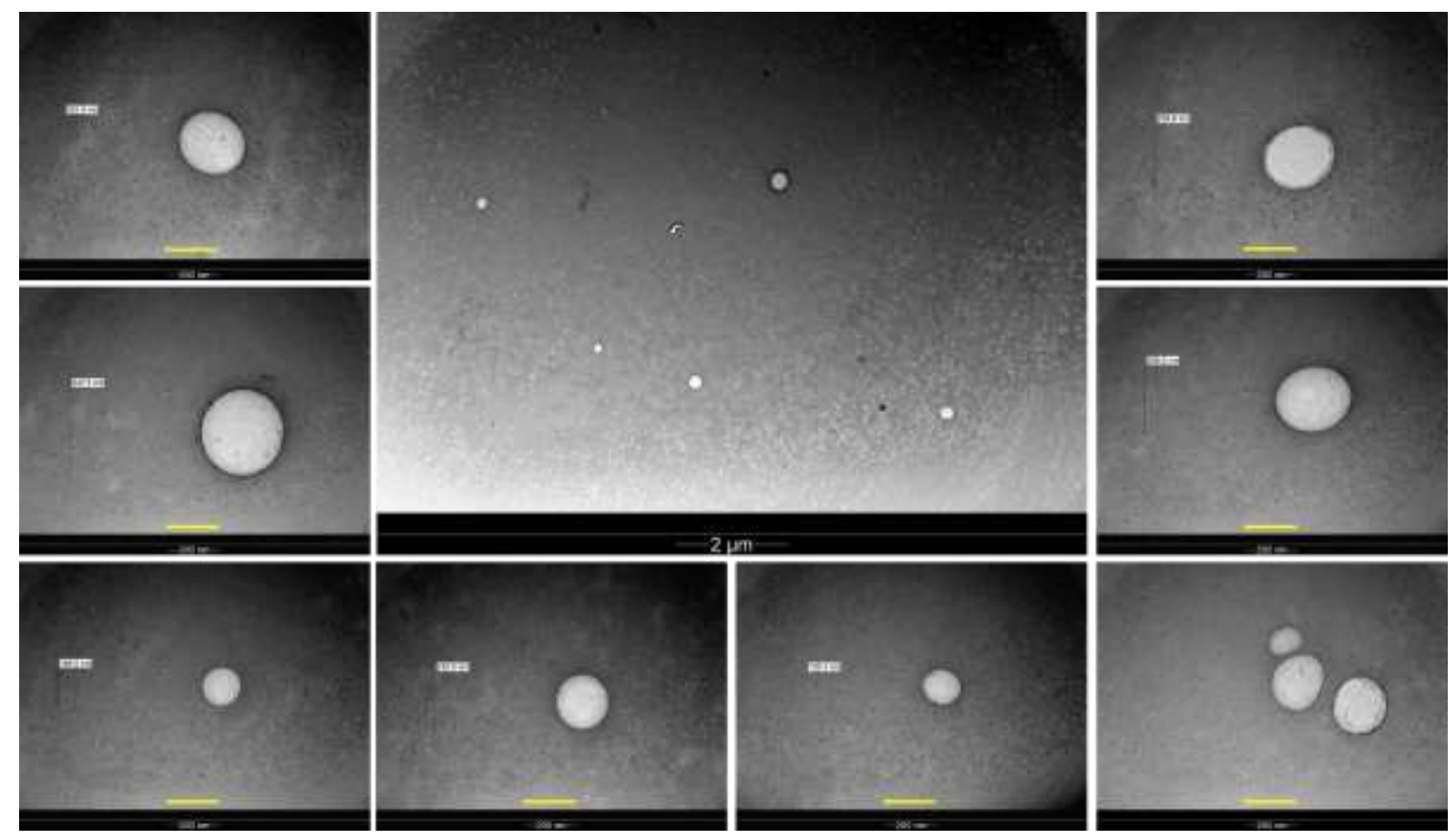

Figure S22. TEM images of the MCF-7 EVs after capture/release process using CD63 aptamerfunctionalized cloth. The bar in all of the TEM images, except the central image, is $200 \mathrm{~nm}$. 


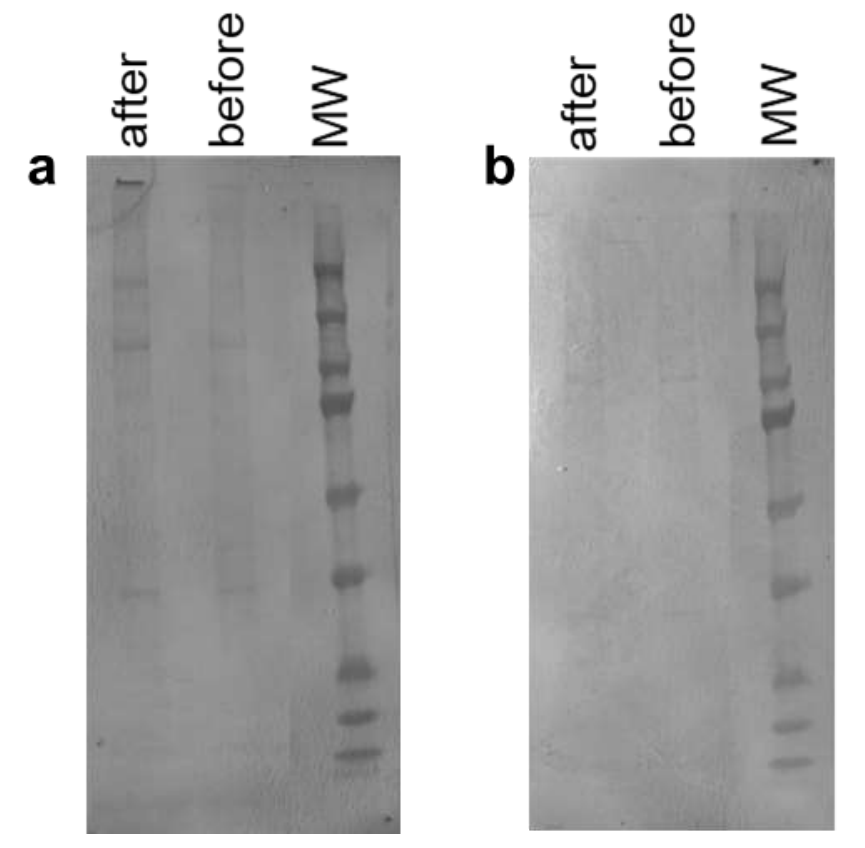

Figure S23. Coomassie blue-stained membranes used for CD63 (a) and CD81 (b) western blots.

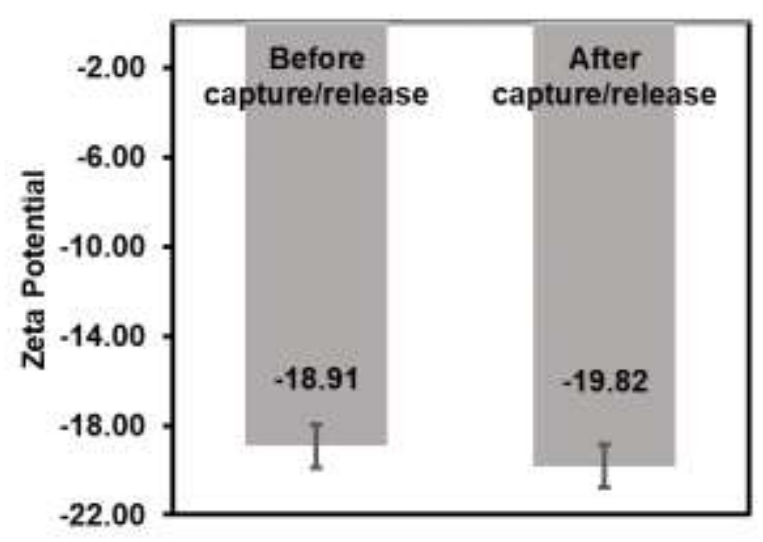

Figure S24. $\zeta$ potential values of the MCF-7 EVs before and after capture/release on the CD63 aptamerfunctionalized cloth $(n=5)$.

\section{References}

(1) Garreau, S., Louarn, G., Buisson, J.P., Froyer, G. \& Lefrant, S. In Situ Spectroelectrochemical Raman Studies of Poly(3,4-ethylenedioxythiophene) (PEDT). Macromolecules 1999, 32, 6807 6812.

(2) Jin, L. et al. A Facile Approach for the Fabrication of Core-Shell PEDOT Nanofiber Mats with Superior Mechanical Properties and Biocompatibility. J. Mater. Chem. B 2013, 1, 1818-1825.

(3) Pistillo, B.R. et al. One Step Deposition of PEDOT Films by Plasma Radicals Assisted Polymerization via Chemical Vapour Deposition. J. Mater. Chem. c 2016, 4, 5617-5625. 
(4) Akbarinejad, A., Ghoorchian, A., Kamalabadi, M. \& Alizadeh, N. Electrospun Soluble Conductive Polypyrrole Nanoparticles for Fabrication of Highly Selective n-Butylamine Gas Sensor. SENSOR ACTUAT B-CHEM 2016, 236, 99-108.

(5) Wang, X., Yang, T., Li, X. \& Jiao, K. Three-Step Electrodeposition Synthesis of Self-Doped Polyaniline Nanofiber-Supported Flower-Like Au Microspheres for High-Performance Biosensing of DNA Hybridization Recognition. Biosens. Bioelectron. 2011, 26, 2953-2959.

(6) Guo, S., Wang, L. \& Wang, E. Templateless, Surfactantless, Simple Electrochemical Route to Rapid Synthesis of Diameter-Controlled 3D Flowerlike Gold Microstructure with "Clean" Surface. CHEM. COMMUN. 2007, 3163-3165.

(7) Lee, K.S. \& Drescher, D.G.J.J.o.B.C. Derivatization of Cysteine and Cystine for Fluorescence Amino Acid Analysis with the o-Phthaldialdehyde/2-Mercaptoethanol Reagent. J. BIOL. CHEM. 1979, 254, 6248-6251.

(8) Roth, M. Fluorescence Reaction for Aamino Acids. Anal. Chem. 1971, 43, 880-882. 